\title{
Sobre reformas y políticas sanitarias en la España de los últimos años del franquismo y la transición democrática
}

\author{
On reforms and health policies in Spain in the final years of the \\ Franco regime and the transition to democracy
}

\author{
Rosa Ballester ${ }^{i}$ \\ i Profesora emérita, Universidad Miguel Hernández. \\ Elche - Alicante - España \\ orcid.org/0000-0002-7870-4185 \\ Rosa.Ballester@umh.es
}

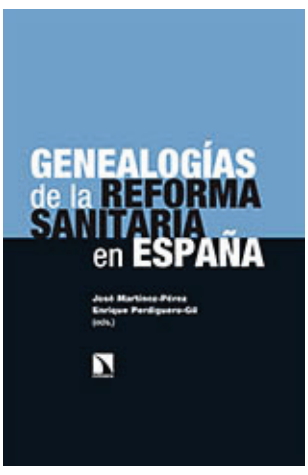

MARTÍNEZ-PÉREZ José; PERDIGUERO-GIL,

Enrique (Ed.). Genealogías de la reforma sanitaria en España. Madrid: Libros de La Catarata. 2020. 304p.
La reforma del sector de la salud hay que entenderla, en sus principios programáticos generales, como un proceso cuyo objetivo es mejorar la equidad, la calidad y la eficiencia mediante cambios en la organización y financiación de los servicios de salud. El análisis histórico de los sistemas sanitarios contemporáneos nos permite valorar y entender el modo como los distintos países se enfrentaron a los formidables retos planteados para hacer frente a la salud y enfermedad de las poblaciones. Este es el núcleo en torno al cual se articula el libro Genealogías de la reforma sanitaria en España, intentando responder a las preguntas del por qué, el cuándo y el cómo tuvo lugar ese proceso en el Estado español de la segunda mitad del siglo XX. El interés de una obra de estas características, coordinada por dos historiadores de reconocida valía y dilatada trayectoria investigadora, reside, en gran medida, en la singularidad de su enfoque, en la potencialidad de las fuentes utilizadas, en la novedad de muchos de sus contenidos, que hacen que su lectura rebase con mucho las fronteras de un estudio país para transformarse en un modelo de trabajo que sin duda va a ser de utilidad a nivel internacional. Examinemos brevemente el contexto político y social en el que el libro se desenvuelve.

Tras el trágico episodio de la Guerra Civil Española (1936-1939), se instauró y tuvo una vigencia de cerca de cuarenta años, la dictadura franquista. A lo largo de este dilatado espacio temporal, hasta la muerte de Francisco Franco en 1975, se fueron sucediendo una serie de cambios, desde el denominado periodo autárquico, caracterizado por el aislacionismo político y social, hasta el fin de dicho aislamiento internacional de España con la entrada del país en los organismos y agencias de las Naciones Unidas, entre ellos, la Organización Mundial de la Salud (OMS) en 1951, en pleno periodo de la Guerra Fría. Los últimos años del régimen franquista, en los que transcurren preferentemente la mayor parte de los 
capítulos del libro, corresponden a la etapa del desarrollismo con la puesta en marcha de los denominados planes de desarrollo económico y social, y el declive del régimen. En todo este contexto se produjeron una serie de cambios que afectaron a la organización sanitaria y que se acentuaron en la etapa denominada de transición democrática, periodo durante el cual el país dejó atrás el régimen dictatorial y pasó a regirse por una constitución (1978) que restauraba la democracia.

En el caso concreto de las transformaciones experimentadas por todos aquellos aspectos referentes a las políticas sanitarias, esta monografía representa una contribución a la tarea de comprender adecuadamente los acontecimientos previos - con iniciativas que no pocas veces estuvieron abocadas al fracaso - a la reforma que delimita el actual ordenamiento sanitario español: la Ley General de Sanidad de 1986. Dicha ley supuso un cambio de gran trascendencia en el modelo político de asistencia sanitaria, que pasó de un sistema de Seguridad Social financiado con las cuotas de trabajadores y empresarios, a un sistema nacional de salud en el que se integraban todas las otras redes existentes y que se financiaba a través de los presupuestos generales del Estado de forma progresiva. Se estableció igualmente la universalidad de la asistencia sanitaria para todos los españoles.

La utilización en el título del plural "genealogías" en lugar de "genealogía" responde a la amplitud y complejidad del tema que, como indican los autores, requeriría de más estudios monográficos de diversos aspectos que permitieran tener una visión de conjunto, global y comprehensiva de la reforma sanitaria en el periodo de tiempo estudiado. Por ello, se ha optado por seleccionar algunos problemas relevantes y significativos por su importante alcance científico, profesional y social. En los tres bloques en los que está estructurado el libro se abordan sucesivamente los problemas de salud en el ámbito rural ("Un mundo en descomposición: la asistencia sanitaria en el mundo rural"), diversas cuestiones de índole educativa y asistencial ("Nuevas respuestas a viejos problemas") y finalmente, análisis de las discusiones en torno a la definición de las fronteras entre la normalidad y la discapacidad con ejemplos prácticos ("Redefiniendo las fronteras de la normalidad").

Médicos y matronas en unos entornos rurales en proceso de despoblación en el periodo del desarrollismo y con los cambios que conllevaron la introducción de nuevas normativas que modificaban las propias figuras profesionales, eran temas escasamente estudiado. La aportación de Ruiz Verdún sobre la asistencia al parto en el mundo rural, el papel desempeñado por las matronas y, como telón de fondo, la medicalización creciente del parto, es muy clarificador e incluye testimonios de estas profesionales junto con otras fuentes. El capítulo de autoría colectiva (Comelles, Perdiguero, Bueno y Barceló), sobre la práctica médica rural durante el franquismo, el de mayor longitud de todo el volumen, merece una mención especial al aunar una pluralidad de fuentes analizadas con rigor, un muy detallado análisis de las peripecias sufridas por el cuerpo de médicos titulares, desde varios puntos de vista, el legislativo, el estructural pero también el modo como percibieron y experimentaron las transformaciones hasta su virtual desaparición con la aparición de la atención primaria a la salud, desde sus propios testimonios. El capítulo está abordado desde la doble perspectiva antropológica e histórica. La presencia de dos de los autores, especialistas en antropología médica e historia (Josep M. Comelles y E. Perdiguero) con una obra muy importante en este terreno (Perdiguero-Gil, 2015; Perdiguero-Gil, Comelles, 
2019), hacen que ese capítulo sea de referencia obligada para el análisis de los médicos rurales - en este entorno español y en el de otros países - y plantea un interesantísimo tema de investigación de largo recorrido en la historiografía: la figura y la percepción social del médico ideal, el que escucha, el que atiende técnicamente pero también el que se ocupa, y se preocupa por sus enfermos de forma altruista y cercana.

En el segundo de los bloques indicados, se articulan cuatro grupos temáticos que tienen en común el formar parte de un grupo de problemas de largo recorrido que experimentaron cambios en su consideración en los años finales del franquismo. David Lorda, un historiador de la psiquiatría, sobre la base de las importantes investigaciones que sobre el particular se están llevando a cabo por autores de la talla de Rafael Huertas, Ricardo Campos o Enric Novella, se ocupa de la asistencia al enfermo mental heredada del franquismo y los intentos de reforma a través de nuevos organismos como el Patronato Nacional de Asistencia Psiquiátrica, desde una perspectiva tecnocrática que no supo estar a la altura de los movimientos de reforma psiquiátrica que en otros países se estaban llevando a cabo.

Dadas las características del régimen franquista, con una fuerte impronta de la moral más conservadora, el tema de la sexualidad en general y de la educación sexual en las escuelas, en particular, era punto menos que conflictivo. Aida Terrón e Inmaculada Hurtado, conocidas expertas en historia de la educación, examinan tanto el contexto internacional de países concretos y de organismos como OMS y Unesco, sobre el tipo y contenidos programáticos de este tipo de educación y la realidad española de los años 1960 y 1970, con las dificultades para conciliar y armonizar los distintos modelos con bases ideológicas plurales, intentando evitar conflictos, en un tema no resuelto.

Pilar León forma parte del grupo de investigación sobre el recorrido histórico en el periodo contemporáneo del hospital, la institución asistencial más emblemática. Su capítulo en este volumen aborda la redefinición del papel jugado por la Iglesia católica en el sistema hospitalario español, en un doble eje de referencia: los cambios asistenciales que afectaron a la red hospitalaria durante el franquismo y la transición y el nuevo marco que supusieron las orientaciones de la Iglesia emanadas del Concilio Vaticano Segundo. Un análisis que incluye desde los cambios en la situación patrimonial de las instituciones existentes, sobre todo las relativas a la infancia, los enfermos mentales y la atención a ancianos y otras patologías residuales, como la lepra, a los cambios cualitativos de tipo profesional e ideológico que afectaron a religiosos y religiosas que tradicionalmente habían sido piezas clave en la estructura de dichas instituciones.

Los problemas y las orientaciones que se llevaron a cabo en el periodo objeto de estudio sobre temas cruciales que afectaban a la propia estructura de las políticas sociales y económicas del país, el de la medicina, la higiene y la seguridad del trabajo, es el contenido del último de los capítulos de este segundo bloque, bajo la autoría de un especialista en estos temas, José Martínez Pérez. A diferencia de otras reformas emprendidas y que se analizan en otros lugares del libro, ésta se orientó siguiendo en gran medida las directrices emanadas de organismos internacionales como la OMS y la Organización Internacional del Trabajo con un discurso que reforzaba la prevención y una visión amplia del concepto de salud en los contenidos de la especialidad de medicina del trabajo, contribuyendo al proceso modernizador que el país estaba experimentando. 
El libro se cierra con un tercer bloque que incluye dos trabajos de Salvador Cayuela y Mercedes del Cura, respectivamente, estudios que se incardinan en el grupo de investigación liderado por el autor del capítulo anterior, con una larga trayectoria en el campo de la historia de las discapacidades. Dos aportaciones con enfoques distintos pero complementarios. El primero, desde perspectivas filosóficas en especial desde el análisis foucaultiano y con la inclusión de un estudio de campo etnográfico, explora el modo cómo las personas con este tipo de problemas fueron gobernadas, conducidas y percibidas por diferentes instancias e instituciones que tenían como objeto la discapacidad y sus protagonistas. El libro se cierra con el capítulo de Del Cura que reconstruye de forma pormenorizada y con un interesante análisis crítico, las políticas relativas a la discapacidad intelectual en las décadas de 1970 y 1980, con referencias a la situación anterior. En este tema fue muy reseñable la influencia de las recomendaciones de expertos de organizaciones sanitarias internacionales sobre las posibilidades que los nuevos conocimientos médicos ofrecían para su prevención y la necesidad del cuidado prenatal, perinatal y postnatal. Las campañas de sensibilización con abundante material y formatos - como cartelismo y uso de los medios de comunicación destinadas a difundir la nueva mirada sobre este tipo de discapacidades - y las normativas y organismos creados específicamente para abordar este tema solo pudieron implementarse y despegar en el entorno político de la transición democrática.

En suma, la monografía constituye una aportación de primer orden al estudio de las reformas sanitarias llevadas a cabo en la segunda mitad del siglo pasado. En primer lugar, por tratarse de una obra que recoge, en manos de un grupo de historiadores de reconocida valía, el resultado de un importante número de investigaciones y trabajos anteriores que permiten aproximarnos, de un modo original y riguroso, a un tema historiográficamente relevante al mostrar los factores que condicionaron estas actuaciones reformistas, alguna de las cuales no llegaron siquiera a cuajar de un modo significativo y cuyo modelo permite realizar análisis comparados posteriores con otros países estableciendo similitudes y diferencias y la importancia de los contextos. En segundo término, porque permite arrojar luz y orientar a los retos actuales de las políticas sanitarias lo que amplía el campo de interés de posibles lectores más allá del campo de la historia.

\section{REFERENCIAS}

MARTÍNEZ-PÉREZ José; PERDIGUERO-GIL, Enrique (Ed.).

Genealogías de la reforma sanitaria en España. Madrid: Libros de La Catarata. 2020.

PERDIGUERO-GIL, Enrique (Ed.). Política, salud y enfermedad en España: entre el desarrollismo y la transición democrática. Elche: Editorial electrónica de la Universidad Miguel Hernández. Epub. Disponible en: <https:// editorial.umh.es/2015/12/09/politica-salud-yenfermedad-en-espana-entre-el-desarrollismo-yla-transicion-democratica/>. Acceso en: 10 mar. 2020. 2015.

PERDIGUERO-GIL, Enrique; COMELLES, Josep

María.

The defence of health: the debates on health reform in 1970s in Spain. Dynamis, v.39, n.1, p.45-72. 2019. 